\section{CNRS strengthens links with industry}

\section{Paris}

ThE Centre National de la Recherche Scientifique (CNRS), the largest of France's state-funded research bodies, has announced the creation of two major research partnerships with industry. The new ventures, one in immunology and the other in metallurgy, reflect the pattern of co-funding with industrial partners being promoted by the present conservative government.

In the fast-changing field of biotechnology, this kind of partnership has obvious mutual benefits in the effort to maintain international competitiveness While state-funded researchers gain access to expensive facilities, the industrial partner is able to tap into the latest developments in molecular biology, often arising from basic research. This was the thinking behind the creation of a joint laboratory by CNRS and the pharmaceutical company bioMérieux.

The new laboratory has been given space at the École Normale Supérieure at Lyons, under the direction of Dr B. Mandrand, of the Department of Immunological Research at bioMérieux. The principal objectives of the collaboration are to develop new in vitro diagnostic techniques and to simplify and automate immunological reactions. Apart from work specifically aimed at the development of early diagnosis of retroviruses, the group will extend an existing collaboration between the CNRS organic materials laboratory and bioMérieux on the development of new reagents.

The joint laboratory, like others already set up with companies such as Saint Gobain, Elf Aquitaine and Rhône-Poulenc, will be funded equally by the partners, initially for 4 years, but a spokeswoman for CNRS would not disclose the budget set aside for the project.

The new Titanium Research Group, announced by CNRS on 1 March, is more diffuse in its organization. Five university metallurgy departments - three of which have CNRS funding - five industrial companies and the government's military research department, DRET, have joined forces in a FF20 million ( $£ 2$ million) effort to promote research into titanium and its alloys.

Last year, research minister Jacques Valade announced his backing for new materials research, but the targeting of titanium alloys, such as TA6V, is a strategic decision. The most immediate application of these alloys is in aerospace industries to which France has a long-standing commitment, both as exporter of military aircraft and manufacturer of space hardware within the European Space Agency.

Peter Coles

\title{
Phillips defends three-tier model for UK academic science
}

\section{London}

THAT British scientists remain deeply divided about the best prescription to cure the nation's ailing science base was clearly demonstrated two weeks ago at a meeting organized by the Society of Chemical Industry (SCI). The present debate was

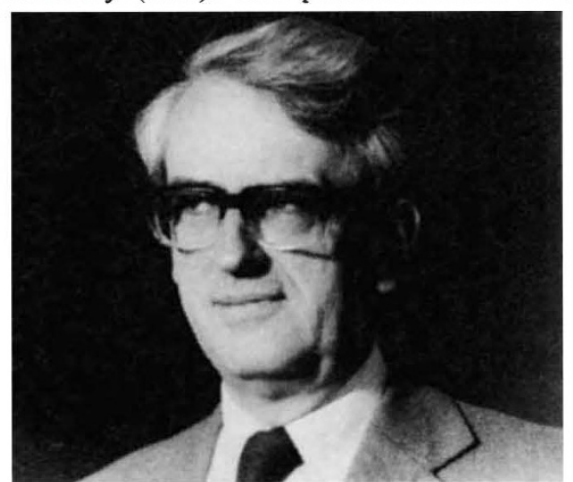

Sir David Phillips-sticking to his guns.

ignited last July with the publication by the Advisory Board for the Research Councils (ABRC) of a discussion document entitled $A$ Strategy for the Science Base (see Nature 328, 280; 1987). The most contentious of the document's 35 recommendations was for differentiation between types of higher education institution engaged in scientific research: type $\mathrm{R}$ (with substantial research activity across all fields); type $\mathrm{X}$ (with substantial research activity in particular fields; and type $\mathrm{T}$ (pursuing the research and scholarship necessary to support teaching but without advanced research facilities). The resulting clamour of opposition from the higher education community has been well documented (see, for example, Nature 330, 3; 1987). But at the SCI meeting, ABRC chairman Sir David Phillips was sticking firmly to his guns.

Phillips seems to have a more sympathetic - or perhaps realistic - view than many of his critics of the government's position on the public financing of science. In the House of Commons debate on 29 February, two days before the meeting, Mr Robert Jackson, the higher education minister, reaffirmed the government's view that the problems facing British science will be solved only by more effective management: "It is simply not possible to bridge the gap between the reach of our scientists and their grasp by increasing public expenditure". Similarly, Phillips says the key to the problem is to learn how to manage the system without depending on continuing growth. "Unless we are to lurch from crisis to crisis, we badly need both a deeper understanding of the dynamics and management of scientific research and the resolution and resources to tackle these problems effec- tively." Furthermore, a year ago $\mathrm{Mr}$ Kenneth Baker, the Secretary of State for Education and Science, made clear his view that research should be concentrated in fewer universities or university departments, possibly to the extent that some universities should become primarily teaching institutions (see Nature 326, 116; 1987).

While welcoming the fact that the ABRC document had appeared to focus the minds of the academic community more sharply on the long-term problems facing science, Phillips feels that much of the opposition to the 'RTX' proposals is based on misconception.

$\mathrm{He}$ accepts that the University Grants Committee is moving some way towards achieving a more streamlined system by its present policy of selectivity, but says that this will not achieve quickly enough the degree of concentration needed. Phillips emphasizes that the RTX categorization was intended to apply to the polytechnics as well as the universities. "Given that not all such institutions are funded for research in the same way at present, there should at least be pause for thought about the potential standing of type $T$ institutions and their role in the developing educational scene." $\mathrm{He}$ "regrets" that the RTX proposals were perceived as introducing a hierarchy. "The difference between a type $\mathrm{R}$ and a type $\mathrm{X}$ institution would have more to do with the range of disciplines in which there was high level research than in the quality and scope of that research. He also stresses that the board did not recommend that differentiation should be imposed from above, "for example by the Secretary of State issuing a list on April 1 1989", but that the institutions themselves should identify their main strengths and future roles.

Phillips decries as a myth the notion that every British university can and does maintain a high level of research activity across a wide range of disciplines. "It is a myth sustained by three factors: first by a belief that university teaching and research are inseparable; second by the opaqueness of the dual support system of university funding; and third by the wish of most universities to retain a presence in as many disciplines as possible."

Responding to Phillips, Professor Ron Hester, of the University of York, contended that the ABRC's proposals offered little hope of attracting the badly needed increase in government support for the science base. "Without that hope the changes envisaged by the ABRC will lead to little advantage for the universities and will not justify the proposed upheaval."

Simon Hadlington 